Article

\title{
New Nanomaterials with Intrinsic Antioxidant Activity by Surface Functionalization of Niosomes with Natural Phenolic Acids
}

\author{
Elisabetta Mazzotta ${ }^{1,2}$, Carla Orlando ${ }^{2}$ and Rita Muzzalupo ${ }^{2, *(D)}$ \\ 1 Centro di Ricerca Olivicoltura, Frutticoltura, Agrumicoltura, Consiglio per la Ricerca in Agricoltura e L'analisi \\ dell'Economia Agraria (CREA-OFA), 87036 Rende, Italy; mazzotta-elisabetta@libero.it \\ 2 Department of Pharmacy, Health and Nutritional Sciences, University of Calabria Via Pietro Bucci, \\ Ed. Polifunzionale, 87036 Arcavacata di Rende, Italy; carla.orlando96@gmail.com \\ * Correspondence: rita.muzzalupo@unical.it; Tel.: +39-0984-493173; Fax: +39-0984-493298
}

Citation: Mazzotta, E.; Orlando, C.; Muzzalupo, R. New Nanomaterials with Intrinsic Antioxidant Activity by Surface Functionalization of Niosomes with Natural Phenolic Acids. Pharmaceutics 2021, 13, 766. https://doi.org/10.3390/

pharmaceutics 13060766

Academic Editor: Carlotta Marianecci

Received: 30 April 2021

Accepted: 20 May 2021

Published: 21 May 2021

Publisher's Note: MDPI stays neutral with regard to jurisdictional claims in published maps and institutional affiliations.

Copyright: (C) 2021 by the authors Licensee MDPI, Basel, Switzerland. This article is an open access article distributed under the terms and conditions of the Creative Commons Attribution (CC BY) license (https:/ / creativecommons.org/licenses/by/ $4.0 /)$.

\begin{abstract}
Nanoantioxidants have emerged as smart devices able to provide improved stability and biocompatibility and sustained and targeted release of conventional antioxidants. In the current research, a new family of nanoantioxidants has been developed by covalently grafting gallic (GA), caffeic (CF) and ferulic (FR) acid on the surfaces of Tween 80 niosomes. First, empty and curcumin (CUR)-loaded vesicles were prepared using a thin-layer evaporation technique and then functionalized with phenolic acids using carbodiimide chemistry. Nanoantioxidants obtained were characterized in terms of size, polydispersity index, zeta potential, and loading efficiency. Their antioxidant activity was studied by ABTS and DPPH assays. Surface functionalization of empty and CUR-loaded vesicles provided stable vesicles with intrinsic antioxidant properties. In vitro antioxidant assays highlighted that vesicles functionalized with FR or GA exhibited better antioxidant activity compared to CF-grafted niosomes. Furthermore, vesicles loaded with CUR and functionalized with GA and CF showed an enhanced scavenging ability of ABTS and DPPH radicals, compared to the single antioxidant-loaded formulations, highlighting an important synergic effect of CUR when used in combination with GA ad CF.
\end{abstract}

Keywords: nanoantioxidant carrier; non-ionic surfactant vesicles; antioxidant grafting; DPPH; synergic therapy

\section{Introduction}

Antioxidants, recognized as prophylactic and therapeutic molecules, are utilized in a number of applications in the pharmaceutical, cosmetic and nutraceutical fields for the countless healthy benefits associated with their use [1]. The involvement of antioxidants in several redox cellular pathways has been intensively studied and further studies are ongoing from scientists to enhance their role of defense against oxidative stress. Oxidative stress based on the overproduction of reactive oxygen species (ROS) is commonly associated with different pathophysiological processes, such as cancer, atherosclerosis, diabetes, Alzheimer's disease, and Parkinson's disease [2-4]. Antioxidants play a critical role in disease prevention and treatment, maintaining a healthy redox balance between proand antioxidant species, thanks to their ability to delay or inhibit the undesired oxidative degradation of biological macromolecules to free radicals.

Despite their huge potential, the application of antioxidants is often limited due to susceptibility to light, oxygen, and $\mathrm{pH}$, poor solubility in physiological fluid, low bioavailability, and improper delivery in undesired cellular compartments [5-7].

In the last decade, nanotechnology has been applied in antioxidant delivery, leading to the development of smart nanocarriers endowed with antioxidant properties, known as 'nanoantioxidants' [8]. Nanoantioxidant systems could overcome many limitations of 
traditional antioxidant molecules and improve their efficacy, thanks to their prolonged stability, improved bioavailability, the ability to avoid fast metabolic clearance, and to provide a controlled and targeted delivery [9].

The free radical scavenging activity of these carriers could be related to the passive delivery of small antioxidant molecules or some intrinsic antioxidant properties of the device. Encapsulation technology is based on the loading of antioxidant molecules in drug delivery systems, such as liposomes [10], niosomes [11], solid lipid nanoparticles [12], and polymeric nanoparticles [13], in order to provide devices able to protect antioxidants and enhance their absorption. On the other hand, some inorganic nanoparticles, such as gold, cerium, and iron nanoparticles, have emerged as devices with intrinsic antioxidant activity related to a radical trapping ability [14].

Another promising strategy involves the surface functionalization of nanocarrier surfaces with small antioxidant molecules to transform inactive devices into antioxidants with improved characteristics. This approach has been actively pursued in recent years and represents an intriguing way not only to provide antioxidant devices, but also to improve several nanocarriers' properties, such as biocompatibility, biostability, and the ability to escape immune system activation [15]. Specifically, the simultaneous loading and functionalization of nanocarriers with antioxidants provides the advantage of delivering high antioxidant amounts and the possibility for the co-delivery of other drugs and, thus, for the use of these devices to exploit any synergic effects [16]. The functionalization of nanodevices with natural antioxidants also allows them to be conferred their specific biological activity, depending on the molecule used for the functionalization process, such as antidiabetic [17], antimicrobial, anticancer [18], and anti-Alzheimer's disease [19]. Considering these advantages, Elle et al. [20] proposed the surface functionalization of mesoporous silica nanoparticles with rutin and caffeic acid as a successful strategy to reduce ROS production and generate safer devices for different pharmaceutical applications. In another study, iron magnetic nanoparticles functionalized with gallic acid exhibited synergistic organicinorganic hybrid antioxidant properties and potent antimicrobial activity on different bacterial and fungal strains [21].

To the best of our knowledge, no studies focusing on niosomes with intrinsic antioxidant properties have been reported in the literature.

In recent years, niosomes, versatile nanosized devices, have been pursued as an increasing research interest for their excellent properties, such as high surface area, high stability, and wide versatility in the composition due to the large availability of non-ionic surfactants [22]. Specifically, the large surface of niosomal vesicles makes them suitable for chemical modifications and offers the possibility to easily customize them to a particular and desired therapeutic application.

Herein, we aimed to synthesize niosomes with intrinsic antioxidant properties by chemical conjugation of phenolic acids onto vesicle surfaces.

Phenolic compounds are secondary plant metabolites and represent the most common molecules with antioxidant activity present in dietary sources, such as vegetables, grapes, red wine, fruit juices, tea, and coffee. These micronutrients, in addition to their potent antioxidant activity, are known to exert a variety of pharmacological effects, such as antiinflammatory, antimicrobial, and anticancer activities [23,24]. It is in our interest, thus, to conjugate these molecules on niosome surfaces, since this could be an efficient strategy to personalize these carriers for a specific therapeutic application and to use these devices as carriers of another drug for a potential combination therapy.

Due to their important antioxidant activity and different biological properties, gallic (GA), ferulic (FR), and caffeic acid (CF) were chosen as antioxidant components of the new nanomaterials. We set out to investigate whether functionalization of these polyphenols onto Tween 80 niosomes' surfaces could provide them with antioxidant properties. After functionalization through chemistry carbodiimide, the developed vesicles were characterized, and their antioxidant activity was evaluated in vitro using 2,2-diphenyl1-picrylhydrazyl (DPPH) and 2,2-azinobis-(3-ethylbenzothiazoline-6-sulfonate) (ABTS) 
assays to investigate the potential of these devices as new antioxidant systems. Furthermore, we also set out to use these systems as carriers of other drugs for a potential synergic therapy. For this purpose, curcumin (CUR), a natural polyphenol widely employed to reduce oxidative stress in many pathologies [25], was loaded into the vesicles' bilayer with the aim of evaluating the potential synergic effects and increased biological activity.

\section{Materials and Methods}

\subsection{Chemicals}

Tween 80, curcumin, gallic acid, ferulic acid, caffeic acid, 2,2-diphenyl-1-picrylhydrazyl (DPPH), 2,2-azinobis-(3-ethylbenzothiazoline-6-sulfonate) (ABTS), and sepharose CL-4B gel were purchased from Sigma-Aldrich (Milan, Italy). All solvents for the high-performance liquid chromatography were used and provided by VWR International Srl (Milan, Italy). All chemicals were used without further purification.

\subsection{Preparation of Niosomes}

Tween 80 multilamellar niosome vesicles (MLVs) were prepared by the traditional thin-film hydration method. In order to obtain CUR-loaded niosomes, $2.7 \times 10^{-6}$ moles of CUR were added to the organic surfactant solution. Small unilamellar vesicles (SUVs) were obtained from MLVs by sonication. The purification method of formulation was gel permeation chromatography. The samples were stored in the dark at $4{ }^{\circ} \mathrm{C}$ until their use in subsequent experiments. CUR entrapment efficiency was then determined using a $\mathrm{UV}$-vis spectrophotometer at $426 \mathrm{~nm}$ and was reported as the percentage of drug loaded into niosomes versus the initial total drug.

Hydrodynamic diameter of niosomes and zeta potential were determined by dynamic/electrophoretic light scattering at $25 \pm 0.1^{\circ} \mathrm{C}$. More details are provided in the Supplementary Materials.

\subsection{Preparation of GA-, CF-, FR-, Conjugated Niosomes}

GA, CF, and FR were chemically grafted onto niosomes' surfaces using EDC/NHS coupling agents (Figure 1) [26]. Briefly, phenolic compounds (0.01 mmol), EDC (0.01 mmol), and NHS (0.01 mmol) were dissolved in ethanol and stirred in an ice bath for $1 \mathrm{~h}$. In the next steps, $0.04 \mathrm{mmol}$ of surfactant mixture was added and incubated overnight at room temperature. Free phenolic compounds and other byproducts (isourea) were removed by dialysis for $4 \mathrm{~h}$.
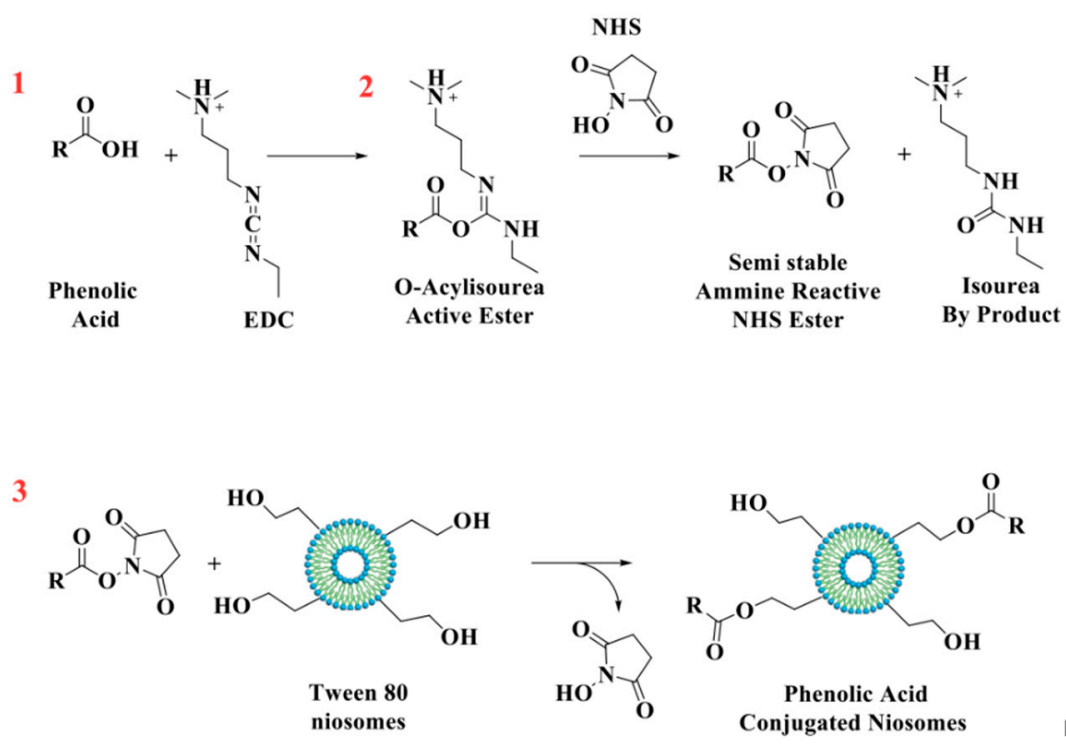

Figure 1. Schematic representation of the reaction between antioxidant molecules and niosomes. 


\subsection{Determination of Total Phenolic Content}

Total phenolic content present in the formulations was evaluated by the Folin-Ciocalteu method [27]. A calibration curve using GA was carried out and the total phenolic content was expressed as GA equivalents (GAE). Grafting efficiency (\%) was determined by measuring the amount of phenolic acids conjugated on the vesicles' surfaces after purification for dialysis, according to the following equation:

$$
\text { Grafting efficiency }(\%)=\frac{\text { phenol content after dialysis }}{\text { phenol content before dialysis }} \times 100
$$

\subsection{DPPH and ABTS Radical Scavenging Activity Assay}

The antioxidant activity was determined by using a DPPH assay according to the method reported by Tavano et al. [11], while the ABTS assay was performed by measuring their capacity to scavenge free radical ABTS [28]. More details are provided in the Supplementary Materials.

\subsection{In Vitro CUR Release Studies}

The CUR release $\left(2.84 \times 10^{-8}\right.$ moles $)$ from antioxidant vesicles was examined under sink conditions in dialysis bags, suspended in $20 \mathrm{~mL}$ of phosphate buffer, $\mathrm{pH}$ 7.4, containing $0.5 \%$ of Tween 80 , under stirring for $24 \mathrm{~h}$ at $37^{\circ} \mathrm{C}$. At specific time points, $2 \mathrm{~mL}$ of the medium was taken and withdrawn with $2 \mathrm{~mL}$ of the fresh buffer. The amount of CUR in the withdrawn samples was analyzed using a UV-vis spectrometer at $426 \mathrm{~nm}$ and determined according to a calibration curve, prepared for the curcumin solutions of known concentrations in the appropriate range [29].

\subsection{Statistical Analysis}

All data were expressed as the mean $\pm \mathrm{SD}$ of three independent experiments. Statistical significance was calculated by one-way analysis of variance (ANOVA) and Bonferronicorrected $p$-value for multiple comparison test. The level of statistically significant difference was defined as $p<0.05$.

\section{Results}

\subsection{Niosomes' Characterization}

In recent years, the design of tailor-made carriers has been actively pursued as a promising way to achieve a personalized therapy. Specifically, functionalization of a nanocarrier surface is an interesting approach used to develop smart devices with specific properties dependent on the molecule used for the grafting process. Niosomes are one of the most used candidates to develop smart nanocarriers for pharmaceutical applications. Indeed, the large availability of functional groups on the hydrophilic surfaces of vesicles allows for easy functionalization.

Herein, novel antioxidant nanodevices were designed through surface functionalization of traditional niosomes with natural polyphenols. Specifically, we selected a Tween 80 surfactant which is rich in -OH terminal groups. Vesicles were prepared and then functionalized using EDC as a coupling agent in order to enhance the reaction of the carboxylic group of phenolic acids with -OH groups on the niosomes' surfaces.

A Tween 80 surfactant resulted in forming stable vesicles of uniform size of around $549.8 \mathrm{~nm}$ and these results are similar to a previous study [30]. Niosomes' sizes were strongly affected by drug loading and surface functionalization, as shown in Figure 2. The conjugation of phenolic acids on the niosomes' surfaces decreased the diameter of niosomes by up to $495.8,433.8,373.1 \mathrm{~nm}$ after conjugation with GA, CF, and FR, respectively (Table 1). 


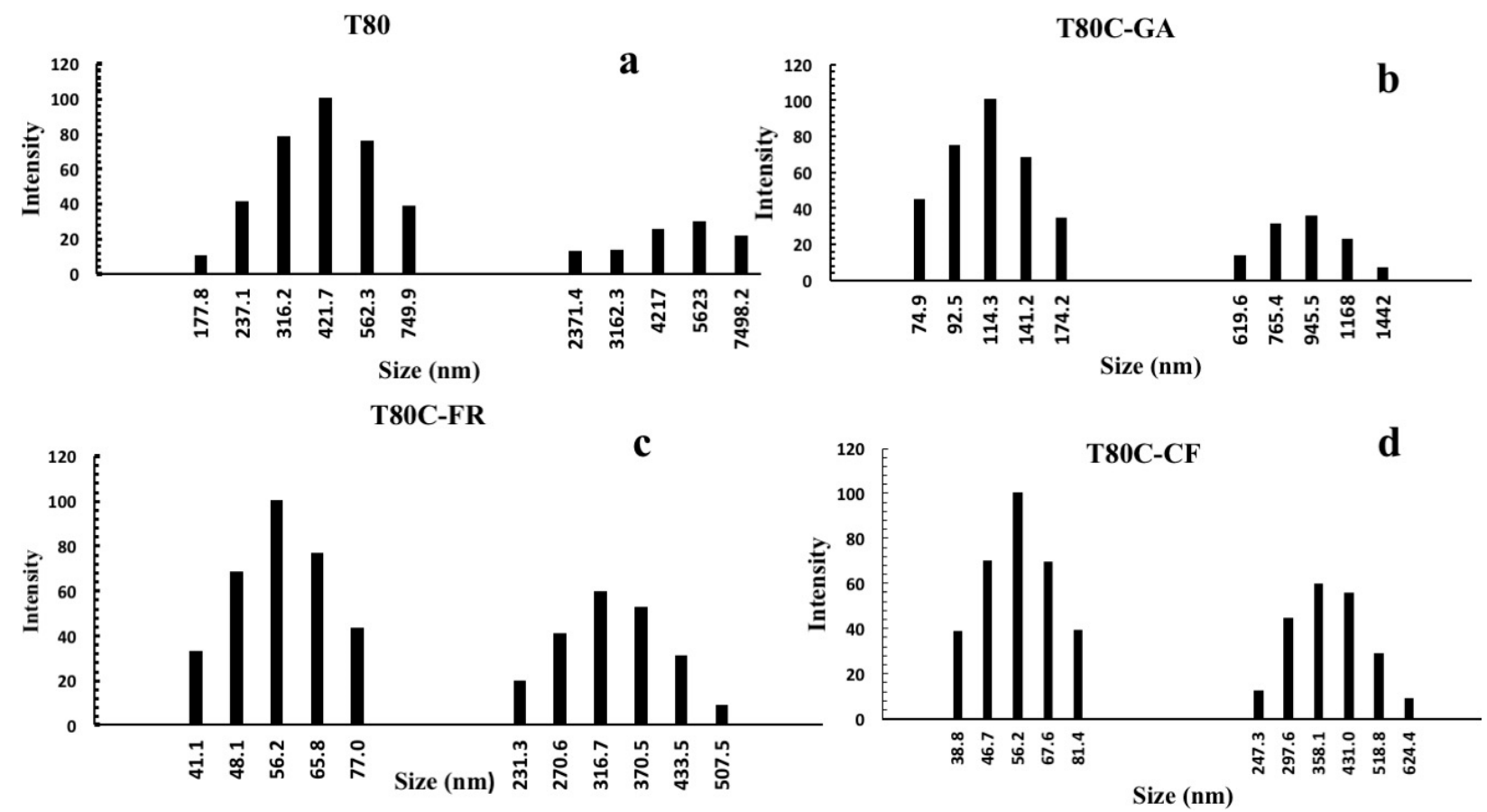

Figure 2. Size distribution of niosomes before (a) and after CUR loading and functionalization with GA (b), FR (c), and CF (d).

Table 1. Average size, polydispersity index (P.I), zeta potential, and grafting efficiency of empty niosomes before and after conjugation with phenolic acids at $25^{\circ} \mathrm{C}$. Data represent the mean \pm S.D. of three independent experiments performed with triplicate measurements.

\begin{tabular}{ccccc}
\hline Formulations & $\begin{array}{c}\text { Size } \\
(\mathbf{n m})\end{array}$ & P.I. & $\begin{array}{c}\text { Zeta Potential } \\
(\mathbf{m} \mathbf{)}\end{array}$ & $\begin{array}{c}\text { Grafting } \\
\text { Efficiency } \\
\mathbf{( \% )}\end{array}$ \\
\hline T80 & $549.8 \pm 16.7$ & $0.265 \pm 0.010$ & $-30.1 \pm 0.577$ & - \\
\hline T80-GA & $495.8 \pm 12.0$ & $0.219 \pm 0.016$ & $-13.4 \pm 0.2$ & $36.1 \pm 4.14$ \\
\hline T80-CF & $433.8 \pm 16.6$ & $0.187 \pm 0.011$ & $-11.1 \pm 0.1$ & $52.5 \pm 0.03$ \\
\hline T80-FR & $373.1 \pm 11.1$ & $0.259 \pm 0.013$ & $-17.6 \pm 0.5$ & $44.9 \pm 7.05$ \\
\hline
\end{tabular}

This could be explained by the hydrophobic nature of phenolic acids and specific interaction occurring between them and the surfactant. Several studies, in fact, showed the formation of intermolecular hydrogen bonds between phenolic compounds and a lipid bilayer as the main cause of the higher membrane cohesion and, subsequently, the reduced vesicle size [31,32]. The phenolic acid/surfactant interactions increased the hydrophobic attraction forces developed among surfactant head-groups, resulting in a smaller surface area for molecules and in a dense and compact structure [33,34]. Another reason could be related to the hydrophobic character of the molecule conjugated on the vesicles' surfaces. In fact, the vesicle size became dependent on the hydrophilic-lipophilic balance of the formulation and the increase in hydrophobicity is commonly associated with the decreased surface-free energy and, consequently, reduced vesicle sizes [35,36]. Furthermore, the decrease in niosome size became dependent on the lipophilicity of phenolic acids conjugated on the vesicles' surfaces: the higher the lipophilicity, the smaller the sizes that were obtained. In fact, the $\log$ P of GA is 0.7, indicating a lower lipophilic character in respect to FR and $\mathrm{CF}$, which present a $\log \mathrm{P}$ equal to 1.67 and 1.53, respectively. Consequently, GA functionalization led to a coating layer on the vesicle with lower cohesion and, consequently, higher sizes in respect to that obtained with FR and CF.

With the aim of evaluating any synergic effect, we loaded in a vesicle bilayer CUR, a natural polyphenol with several reported possibilities in the treatment of different diseases, 
such as cancer, inflammation, arthritis, and metabolic disorders [37]. The pharmacological effects of this compound are associated with its strong antioxidant activity and different studies have indicated an important antioxidant synergic effect when used in combination with GA [38], resveratrol [39], and quercetin [40].

In light of our results, we decided to investigate whether the combination of CUR and the new antioxidant niosomes could provide similar results.

Relevantly, the loading of the drug affected the vesicle sizes (Table 2) (Figure 2). Due to its lipophilic nature, CUR is completely embedded in the lipid bilayer and forms hydrogen bonds with surfactant molecules that results in a decrease in the vesicle sizes. In the literature, the similarity of CUR to cholesterol as a condensing agent on the vesicle membrane has been widely reported [41,42]. Cholesterol results in increased membrane packing, leading to a reduced size of niosomal formulations $[43,44]$. In any case, this smaller size is a desirable feature for drug delivery systems since it is a crucial factor influencing several parameters, such as better stability in vivo, extended bioavailability, reduced selective uptake by the reticuloendothelial system (RES), and the ability to deliver drugs to targets that are difficult to reach $[45,46]$.

Table 2. Physicochemical properties (hydrodynamic diameter, polydispersity index (P.I.), and zeta potential) of curcumin-loaded niosomes before and after conjugation with phenolic acids at $25{ }^{\circ} \mathrm{C}$. Data represent the mean \pm S.D. of three independent experiments performed with triplicate measurements.

\begin{tabular}{ccccc}
\hline Formulations & $\begin{array}{c}\text { Size } \\
\mathbf{( n m )}\end{array}$ & P.I. & $\begin{array}{c}\text { Zeta Potential } \\
\mathbf{( m V )}\end{array}$ & $\begin{array}{c}\text { Grafting } \\
\text { Efficiency } \\
\mathbf{( \% )}\end{array}$ \\
\hline T80C & $201.25 \pm 9.32$ & $0.251 \pm 0.036$ & $-27.8 \pm 0.283$ & - \\
\hline T80C-GA & $91.70 \pm 4.50$ & $0.225 \pm 0.042$ & $-13.3 \pm 1.04$ & $14.6 \pm 3.15$ \\
\hline T80C-CF & $83.70 \pm 2.60$ & $0.273 \pm 0.007$ & $-14.9 \pm 0.850$ & $50.8 \pm 0.09$ \\
\hline T80C-FR & $72.5 \pm 1.11$ & $0.281 \pm 0.018$ & $-15.9 \pm 0.404$ & $41.4 \pm 5.07$ \\
\hline
\end{tabular}

The polydispersity index of the samples ranged from 0.187 to 0.281 , indicating the good homogeneity and quality of niosomal formulations. All developed nanosystems were, in the long term, stable, and any creaming, sedimentation, and flocculation was observed after 3 months of storage in the dark at room temperature.

CUR was successfully entrapped in the niosomal bilayer: in particular, $7.1 \times 10^{-7}$ moles of the drug, corresponding to approximately $26.30 \%$, were loaded and any drug leakage was observed during the functionalization process.

The Folin-Ciocalteu method was used to determine the total amount of phenolic acids grafted onto the niosomal surface using GA as a standard. The amount of polyphenol grafted on the niosomal surface was $0.11,0.19$, and $0.15 \mathrm{mg} \mathrm{GAE} / \mathrm{mL}$ for T80-GA, T80-CF, and T80-FR, respectively.

Grafting efficiency was further assessed using UV-vis spectroscopy and the data obtained (Table 1) confirmed the results obtained with the Folin-Ciocalteu method.

\subsection{Antioxidant Activity}

In order to investigate whether the chemical conjugation of phenolic acids on the niosomal surface provided them with antioxidant activity, DPPH and ABTS assays were performed.

A DPPH test is an easy and fast method that involves the reduction of DPPH $\bullet$ radicals in the non-radical form, resulting in a change in the color of the solution from purple to yellow. First, the antioxidant activity of antioxidant vesicles alone was evaluated at different concentrations, and then in the presence of an amount of CUR equal to that entrapped in the vesicle bilayer. 
The antioxidant activity of empty and un-functionalized niosomes was also studied and the obtained results confirmed that the vesicle based on the Tween 80 surfactant did not show intrinsic scavenging activity (data not shown).

The percentages of the DPPH scavenging activity of niosomes in the functionalization of antioxidant moles grafted onto the vesicle surfaces are reported in Figure 3. All niosomal formulations showed a dose-dependent DPPH scavenging activity.

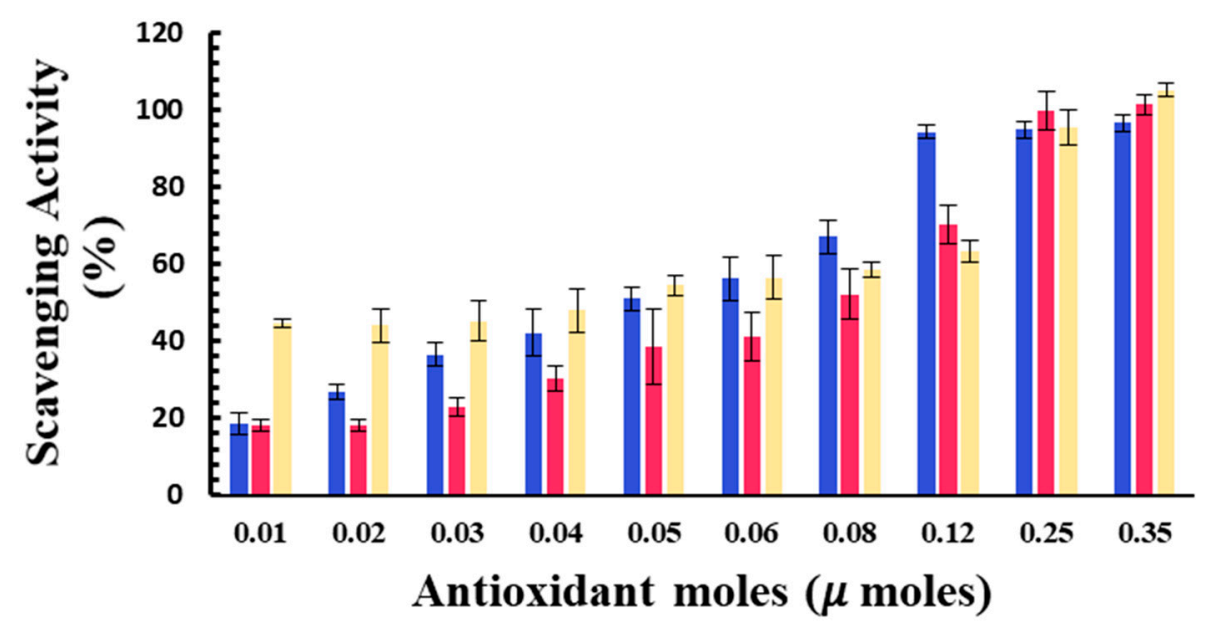

\section{$\square$ T80 GA $\quad$ T80 CF $\square$ T80 FR}

Figure 3. Antioxidant activity of T80-GA (blue), T80-CF (red), and T80-FR (yellow) against DPPH• expressed as a percentage of radical scavenging activity versus antioxidant $\mu$ moles grafted onto niosomes' surfaces. Results represent the mean \pm S.D. of three independent experiments performed with triplicate measurements.

Data reported in Figure 3 show that the covalent grafting of phenolic acids on a niosome's surface represent a successful strategy to develop nanomaterials with intrinsic antioxidant properties. To the best of our knowledge, this study provides the first proof of concept of niosomes with intrinsic free radical scavenging properties that could be used as versatile platforms for pharmaceutical, cosmetic, and nutraceutical applications.

The antioxidant activity of the designed formulation was compared considering the $50 \%$ of inhibition of DPPH radicals in the functionalization of the moles of antioxidants grafted onto niosomes' surfaces (SC50).

Better antioxidant activity was observed for T80-FR and T80-GA, as indicated by their SC50 values equating to 0.041 and $0.051 \mu$ moles, respectively (Table 3). On the contrary, the DPPH scavenging ability of T80-CF was lower, in respect to the other formulations: in fact, the higher value of SC50 equating to $0.087 \mu$ moles highlighted a reduced ability to scavenge free radicals. For the vesicles functionalized with $0.03 \mu$ moles of phenolic acids on their surfaces, the scavenging activity against DPPH radicals, indeed, was $45.18 \%$ and $36.47 \%$, respectively, for T80-FR and T80-GA, while for T80-CF, it was only approximately $22.83 \%$.

Furthermore, we decided to enhance the antioxidant activity of the designed vesicles through the combination with CUR in order to exploit any potential synergic effect. The loading of CUR in the vesicle bilayers led to different responses, depending on the phenolic acids conjugated on the vesicles' surfaces. In particular, data obtained for T80C-FR niosomes showed that CUR loading in the niosomal bilayer led to a reduced response. At the lowest concentration tested, indeed, the DPPH scavenging activity for T80C-FR was $23.16 \%$ against the $44.49 \%$ obtained for T80-FR. This trend could be due to a potential antagonistic effect of FR and CUR. On the contrary, a synergic effect for T80C-GA and T80C-CF was observed. In fact, the antioxidant activity of T80C-GA and T80C-CF was higher in respect to the sum of the effects of the singular components (Figure 4). For instance, at the lowest concentration tested, the scavenger ability of T80C-GA was $44.89 \%$, against the percentage 
obtained with T80-GA and T80C, equating to $18.46 \%$ and $16.44 \%$, respectively. In fact, samples loaded with CUR and externally grafted with CF and GA showed SC50 values that were statistically different $(p<0.05)$ in respect to the formulations loaded only with one antioxidant component (Table 3). The reduction in SC50 values suggested a better antioxidant activity when phenolic acid-grafted niosomes were used in combination with CUR, compared to the corresponding single-loaded formulations.

Table 3. SC50 values of antioxidant-functionalized niosomes in DPPH and ABTS scavenging assay. Results represent the mean \pm S.D. of three independent experiments performed with triplicate measurements. ${ }^{*} p<0.05$ phenolic acid-conjugated T80 niosomes vs. phenolic acid-conjugated T80C niosomes.

\begin{tabular}{ccc}
\hline Sample & $\begin{array}{c}\text { SC50 for DPPH } \\
(\mu \text { moles })\end{array}$ & $\begin{array}{c}\text { SC50 for ABTS } \\
(\mu \text { moles })\end{array}$ \\
\hline T80-FR & $0.041 \pm 0.004$ & $0.014 \pm 0.1$ \\
\hline T80C-FR & $0.041 \pm 0.004$ & $0.013 \pm 0.1$ \\
\hline T80-GA & $0.051 \pm 0.005^{*}$ & $0.014 \pm 0.1^{*}$ \\
\hline T80C-GA & $0.021 \pm 0.002$ & $0.0057 \pm 0.0006$ \\
\hline T80-CF & $0.087 \pm 0.009^{*}$ & $0.025 \pm 0.002$ \\
\hline T80C-CF & $0.047 \pm 0.005$ & $0.026 \pm 0.003$ \\
\hline
\end{tabular}

According to the results, CUR increased the antioxidant activity of T80-GA and T80-CF vesicles; this synergic effect allows its use to lower the amount of active substance required, while maintaining its antioxidant efficacy in clinical practice.

Considering the multiple mechanisms involved in the antioxidant effect, a single test is not enough to evaluate the antioxidant capacities of the new materials. Consequently, we also performed an ABTS assay to obtain deeper insights. Polyphenolic acids retained their ABTS radical scavenging activity after conjugation on the niosomes' surfaces (Figure 5). The results obtained with the ABTS assay were quite different compared to the DPPH assay since the formulations were more sensitive to the test. A higher antioxidant activity was recorded at the lowest concentration of antioxidants grafted onto the vesicles' surfaces. In fact, SC50 values against ABTS free radicals were significantly lower in respect to the SC50 values against the DPPH radicals, indicating a better scavenging ability.

Both T80-GA and T80-FR with an SC50 of $0.014 \mu$ moles exhibited better antioxidant ABTS radical scavenging activity than T80-CF (Table 3). These results were consistent with the DPPH assay, and also confirmed the synergic effect of CUR and GA: in fact, as indicated by the SC50 values reported in Table 3, T80C-GA was found to have an SC50 of $0.0057 \mu$ moles, whereas T80-GA showed an SC50 of $0.014 \mu$ moles and these data were statistically different. This synergic effect allows the use of lower amounts of antioxidants to obtain an enhanced radical scavenging efficacy. These results highlighted the possibility to use CF or GA and CUR together to obtain devices with enhanced therapeutic potential in different oxidative stress-related diseases. Nonetheless, no synergic effect was observed when CUR was loaded onto T80-CF niosomes.

Finally, these results indicated that the conjugation of phenolic acids on niosome surfaces provides them with antioxidant activity and thus makes it possible to use these devices for the treatment of pathologies related to free radicals. The obtained nanomaterials in which one phenolic moiety is exposed in the outer surface and a second antioxidant molecule is loaded onto the vesicle bilayer also showed better antioxidant activity in respect to the devices endowed with a single antioxidant component. This suggests the great potential of these nanodevices as promising tools for the treatment of diseases caused by oxidative stress through combined approaches. 
GALLIC ACID NIOSOMES

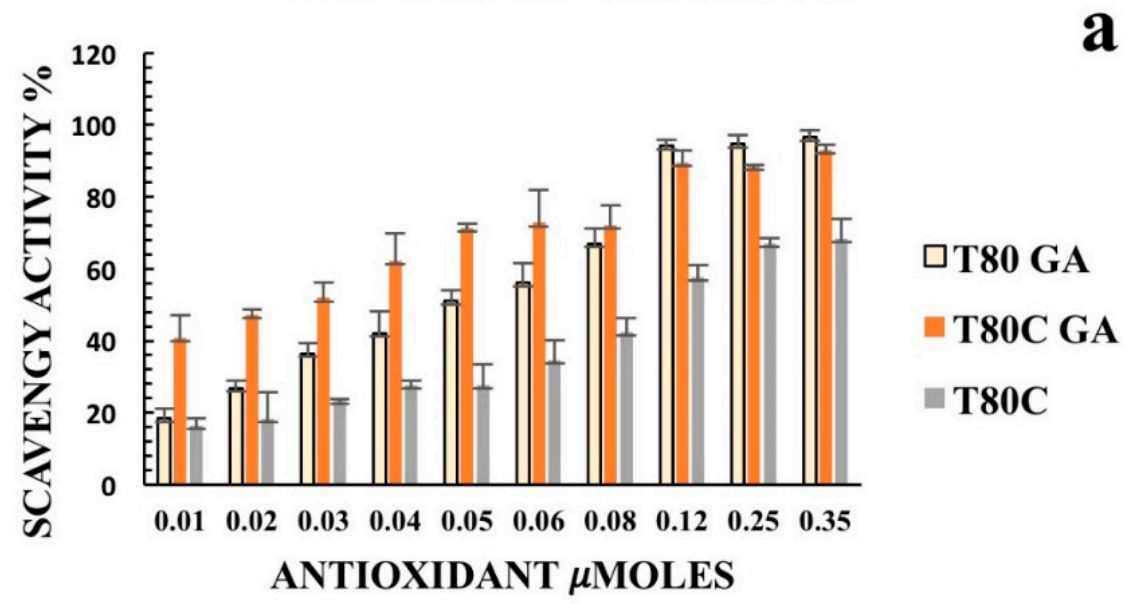

FERULIC ACID NIOSOMES
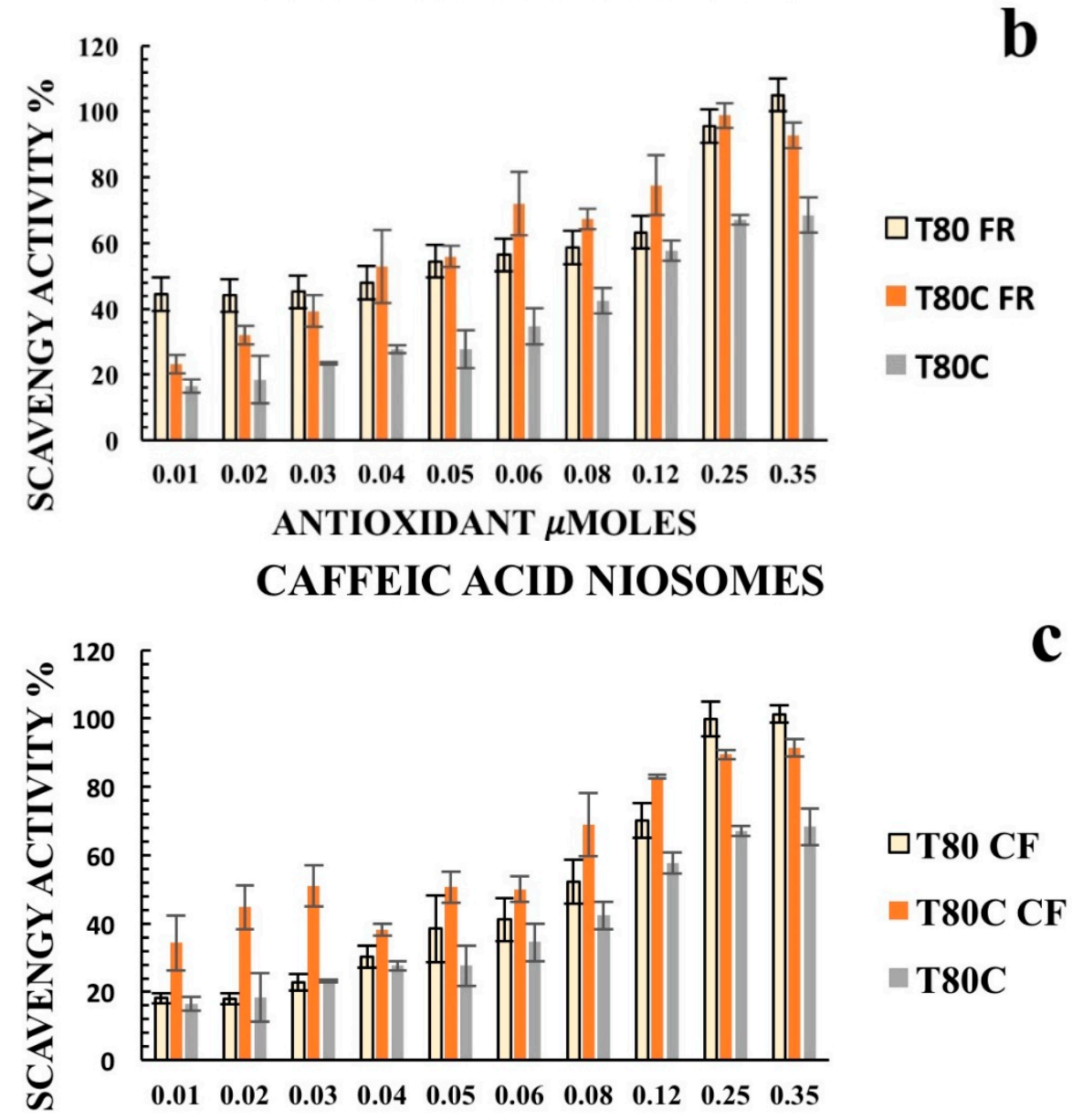

ANTIOXIDANT $\mu$ MOLES

Figure 4. Antioxidant activity of empty (yellow) and CUR-loaded (orange) T80-GA (a), T80-FR (b), and $\mathrm{T} 80-\mathrm{CF}(\mathbf{c})$ against $\mathrm{DPPH} \bullet$ expressed as a percentage of radical scavenging activity versus antioxidant $\mu$ moles grafted on the niosomes' surfaces. Data represent the mean \pm S.D. of three independent experiments performed with triplicate measurements. 


\section{GALLIC ACID NIOSOMES}

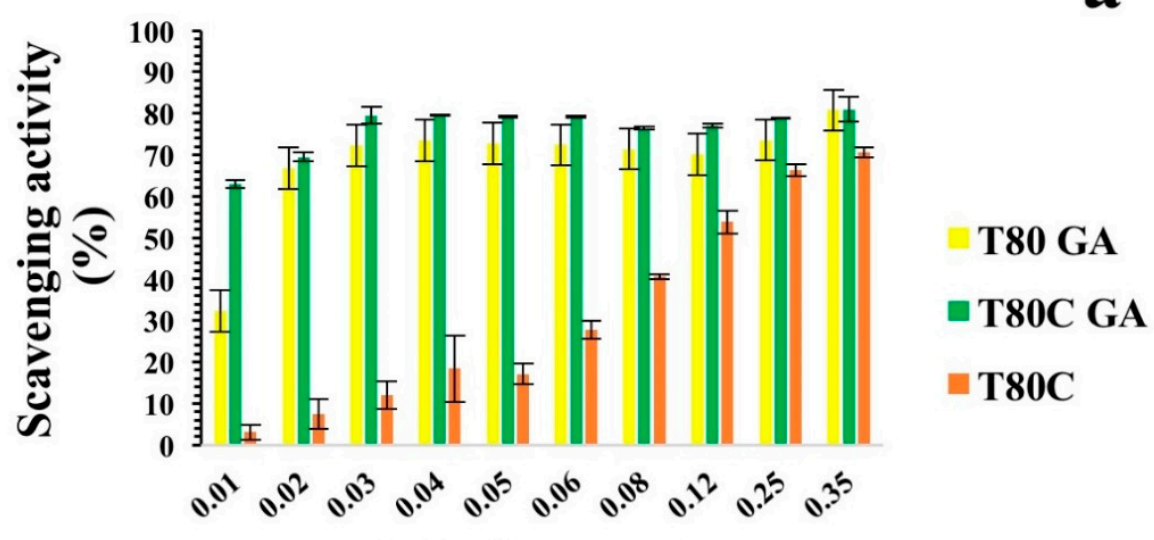

Antioxidant $\mu$ moles

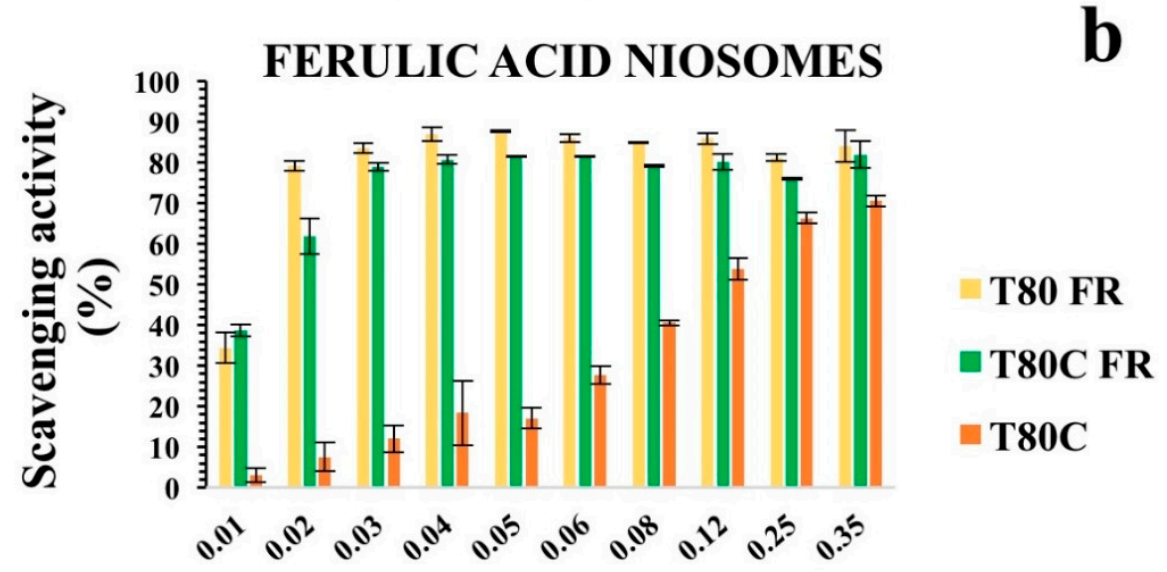

Antioxidant $\mu$ moles

\section{CAFFEIC ACID NIOSOMES}

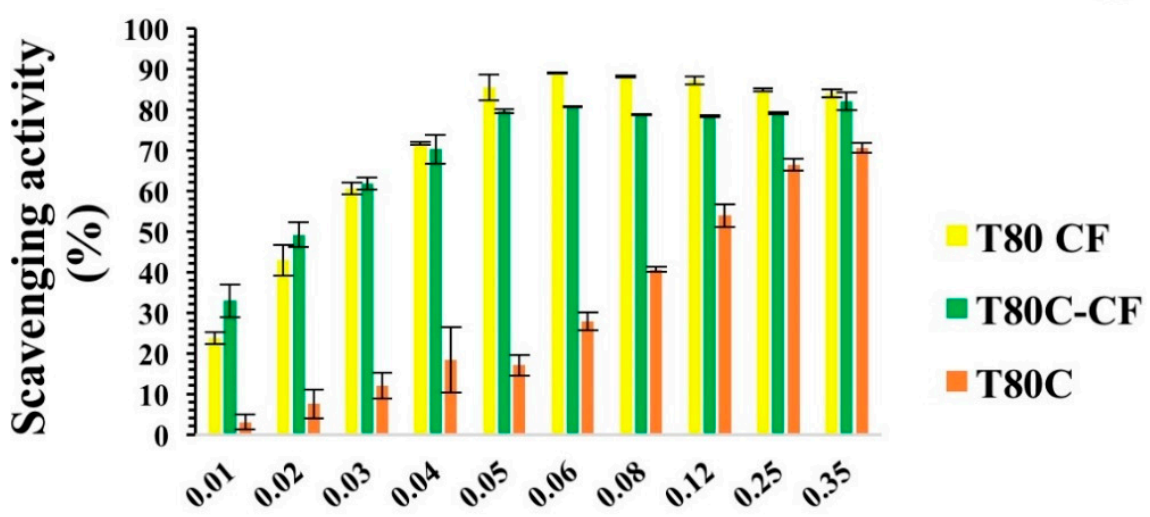

\section{Antioxidant $\mu$ moles}

Figure 5. Antioxidant activity of empty (yellow) and CUR-loaded (orange) T80-GA (a), T80-FR (b), and T80-CF; (c) against ABTS• expressed as percentage of radical scavenging activity versus antioxidant $\mu$ moles grafted onto niosomes' surfaces. Results represent the mean \pm S.D. of three independent experiments performed with triplicate measurements. 


\subsection{In Vitro Release Studies}

In vitro drug release profiles of CUR from vesicles are shown in Figure 6. The release of free CUR was used as a control and the total amount of drug was released in $4 \mathrm{~h}$. As expected, CUR release from niosome samples was retarded, compared to that obtained by a drug-free solution. These results indicated that the designed vesicles can control and sustain the CUR release in time, suggesting the role of these devices as delayed delivery systems. FR- and CF-functionalized systems showed similar drug release trends. On the contrary, the release from T80C-GA was the highest, achieving $78 \%$ of the drug released after $24 \mathrm{~h}$. These different patterns could be ascribed to the different lipophilicity of the phenolic acids conjugated on the vesicle surfaces. As previously mentioned, the hydrophobicity of molecules conjugated on the vesicle surfaces affected the size. Probably, the presence of GA, with a lower hydrophobic character, on the niosomal surface led to the formation of a layer with lower cohesion and, consequently, a higher drug diffusion rate in respect to the layer formed by FR and CF.

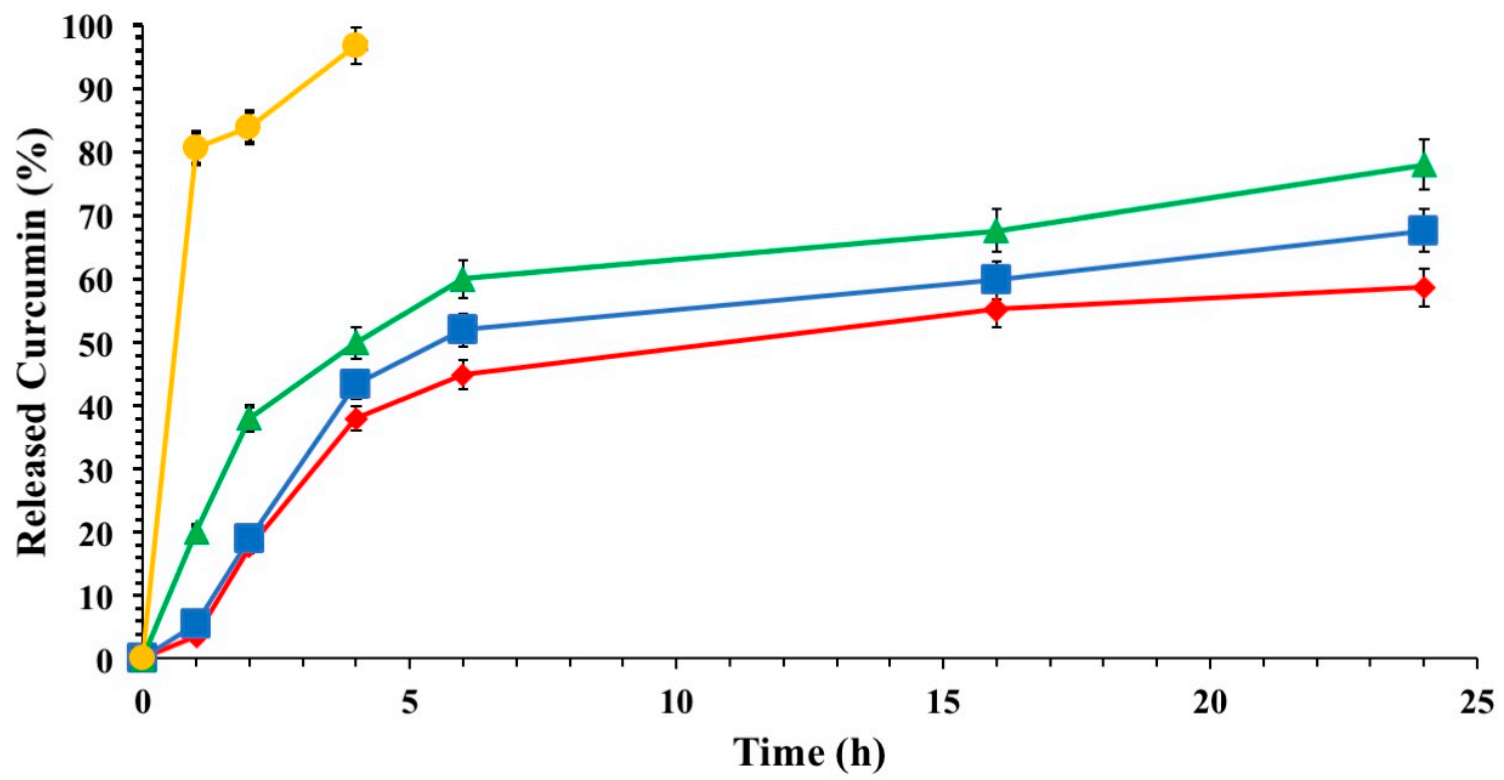

\section{$\rightarrow$ T80C-CF $=$ T80C-FR $₫$ T80C-GA CUR}

Figure 6. In vitro CUR release in phosphate buffer pH 7.4 (Tween $800.5 \%$ ) at $37{ }^{\circ} \mathrm{C}$ from T80C-GA (ム), T80C-CF ( $)$ ), T80C-FR ( $\mathbf{\square})$, CUR solution $(\bullet)$. In all cases, each value represents the mean \pm S.D. of three independent experiments.

\section{Conclusions}

In this work, we prepared the first example of niosomes with intrinsic antioxidant activity by chemical conjugation of phenolic acids onto the vesicle surface.

The data obtained confirmed our hypothesis that surface functionalization of niosomes provides them with antioxidant activity and offers the possibility of using these devices for the treatment and prevention of diseases associated with oxidative stress. Moreover, these vesicles may be used as carriers of other biologically active molecules for multiple and synergic drug therapy. Curcumin, loaded in these formulations, was used as a model drug and the results highlighted an important synergic antioxidant effect.

Supplementary Materials: The following are available online at https:/ / www.mdpi.com/article / 10.3390/pharmaceutics13060766/s1.

Author Contributions: E.M. and C.O. conducted the experiments. R.M. and E.M. designed the experiments and proofread the manuscript. All authors were involved in writing and approving the final manuscript. All authors have read and agreed to the published version of the manuscript. 
Funding: This research received no external funding.

Institutional Review Board Statement: Not applicable.

Informed Consent Statement: Not applicable.

Data Availability Statement: All data presented in this study are included within the article.

Acknowledgments: This research was supported by M.U.R.S.T. (Italy).

Conflicts of Interest: The authors declare no conflict of interest.

\section{References}

1. Rajat, S.; Aarti, Y.; Aditya, S.; Nitin, S. Nano-antioxidants: An emerging strategy for intervention against neurodegenerative conditions. Neurochem. Int. 2015, 89, 209-226.

2. Koltover, V. Antioxidant biomedicine: From free radical chemistry to systems biology mechanisms. Russ. Chem. Bull. 2010, 59, 37-42. [CrossRef]

3. Masoudkabir, F.; Sarrafzadegan, N.; Gotay, C.; Ignaszewski, A.; Krahn, A.D.; Davis, M.K.; Franco, C.; Mani, A. Cardiovascular disease and cancer: Evidence for shared disease pathways and pharmacologic prevention. Atherosclerosis 2017, 263, 343-351. [CrossRef] [PubMed]

4. Liguori, I.; Russo, G.; Curcio, F.; Bulli, G.; Aran, L.; Della-Morte, D.; Gargiulo, G.; Testa, G.; Cacciatore, F.; Bonaduce, D.; et al. Oxidative stress, aging, and diseases. Clin. Interv. Aging 2018, 13, 757-772. [CrossRef] [PubMed]

5. Valgimigli, L.; Baschieri, A.; Amorati, R. Antioxidant activity of nanomaterials. J. Mater. Chem. B 2018, 6, 2036-2051. [CrossRef] [PubMed]

6. Souto, E.B.; Severino, P.; Basso, R.; Santana, M.H. Encapsulation of antioxidants in gastrointestinal-resistant nanoparticulate carriers. Methods Mol. Biol. 2013, 1028, 37-46.

7. Milinčić, D.D.; Popović, D.A.; Lević, S.M.; Kostić, A.Ž.; Tešić, Ž.L.; Nedović, V.A.; Pešić, M.B. Application of Polyphenol-Loaded Nanoparticles in Food Industry. Nanomaterials 2019, 9, 1629. [CrossRef]

8. Khalil, I.; Yehye, W.A.; Etxeberria, A.E.; Alhadi, A.A.; Dezfooli, S.M.; Julkapli, N.B.M.; Basirun, W.J.; Seyfoddin, A. Nanoantioxidants: Recent trends in antioxidant delivery applications. Antioxidants 2020, 9, 24. [CrossRef]

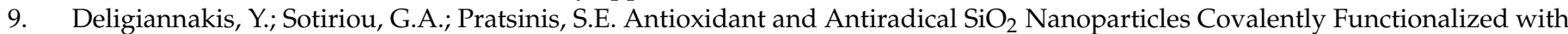
Gallic Acid. ACS Appl. Mater. Interfaces 2012, 4, 6609-6617. [CrossRef]

10. Yuan, J.J.; Qin, F.G.; Tu, J.L.; Li, B. Preparation, characterization, and antioxidant activity evaluation of liposomes containing water-soluble hydroxytyrosol from olive. Molecules 2017, 22, 870. [CrossRef]

11. Tavano, L.; Muzzalupo, R.; Picci, N.; de Cindio, B. Co-encapsulation of lipophilic antioxidants into niosomal carriers: Percutaneous permeation studies for cosmeceutical applications. Colloids Surf. B 2014, 114, 144-149. [CrossRef] [PubMed]

12. Gokce, E.H.; Korkmaz, E.; Dellera, E.; Sandri, G.; Bonferoni, M.C.; Ozer, O. Resveratrol-loaded solid lipid nanoparticles versus nanostructured lipid carriers: Evaluation of antioxidant potential for dermal applications. Int. J. Nanomed. 2012, 7, 1841. [CrossRef] [PubMed]

13. Xie, X.; Tao, Q.; Zou, Y.; Zhang, F.; Guo, M.; Wang, Y.; Wang, H.; Zhou, Q.; Yu, S. PLGA nanoparticles improve the oral bioavailability of curcumin in rats: Characterizations and mechanisms. J. Agric. Food Chem. 2011, 59, 9280-9289. [CrossRef] [PubMed]

14. BarathManiKanth, S.; Kalishwaralal, K.; Sriram, M.; Pandian, S.R.K.; Youn, H.-S.; Eom, S.; Gurunathan, S. Anti-oxidant effect of gold nanoparticles restrains hyperglycemic conditions in diabetic mice. J. Nanobiotechnol. 2010, 8, 16. [CrossRef]

15. Marrazzo, P.; O'Leary, C. Repositioning natural antioxidants for therapeutic applications in tissue engineering. Bioengineering 2020, 7, 104. [CrossRef]

16. Massaro, M.; Riela, S.; Guernelli, S.; Parisi, F.; Lazzara, G.; Baschieri, A.; Amorati, R. A synergic nanoantioxidant based on covalently modified halloysite-trolox nanotubes with intra-lumen loaded quercetin. J. Mater. Chem. B 2016, 4, $2229-2241$. [CrossRef]

17. Liu, J.; Lu, J.F.; Kan, J.; Jin, C.H. Synthesis of chitosan-gallic acid conjugate: Structure characterization and in vitro anti-diabetic potential. Int. J. Biol. Macromol. 2013, 62, 321-329. [CrossRef]

18. Hu, B.; Wang, Y.; Xie, M.; Hu, G.; Ma, F.; Zeng, X. Polymer nanoparticles composed with gallic acid grafted chitosan and bioactive peptides combined antioxidant, anticancer activities and improved delivery property for labile polyphenols. J. Funct. Foods 2015, 15, 593-603. [CrossRef]

19. Eom, T.K.; Ryu, B.; Lee, J.K.; Byun, H.G.; Park, S.J.; Kim, S.K. $\beta$-Secretase inhibitory activity of phenolic acid conjugated chitooligosaccharides. J. Enzym. Inhib. Med. Chem. 2013, 28, 214-217. [CrossRef]

20. Elle, R.E.; Rahmani, S.; Lauret, C.; Morena, M.; Bidel, L.P.R.; Boulahtouf, A.; Balaguer, P.; Cristol, J.P.; Durand, J.P.; Charnay, C.; et al. Functionalized Mesoporous Silica Nanoparticle with Antioxidants as a New Carrier That Generates Lower Oxidative Stress Impact on Cells. Mol. Pharm. 2016, 13, 2647-2660. [CrossRef] 
21. Shah, S.T.; Yehye, W.A.; Saad, O.; Simarani, K.; Chowdhury, Z.Z.; Alhadi, A.A.; AlAni, L.A. Surface Functionalization of Iron Oxide Nanoparticles with Gallic Acid as Potential Antioxidant and Antimicrobial Agents. Nanomaterials 2017, 7, 306. [CrossRef] [PubMed]

22. Muzzalupo, R.; Mazzotta, E. Do niosomes have a place in the field of drug delivery? Expert Opin. Drug Deliv. 2019, 16, 1145-1147. [CrossRef]

23. Dzah, C.S.; Duan, Y.; Zhang, H.; Wen, C.; Zhang, J.; Chen, G.; Ma, H. The effects of ultrasound assisted extraction on yield, antioxidant, anticancer and antimicrobial activity of polyphenol extracts: A review. Food Biosci. 2020, 35, 100547. [CrossRef]

24. Alam, M.A. Anti-hypertensive Eect of Cereal Antioxidant Ferulic Acid and Its Mechanism of Action. Front. Nutr. 2019, 6, 121. [CrossRef]

25. Rahmani, A.H.; Alsahli, M.A.; Aly, S.M.; Khan, M.A.; Aldebasi, Y.H. Role of curcumin in disease prevention and treatment. Adv. Biomed. Res. 2018, 7, 38. [CrossRef] [PubMed]

26. Tavano, L.; Mauro, L.; Naimo, G.D.; Bruno, L.; Picci, N.; Ando, S.; Muzzalupo, R. Further Evolution of Multifunctional Niosomes Based on Pluronic Surfactant: Dual Active Targeting and Drug Combination Properties. Langmuir 2016, 32, 8926-8933. [CrossRef] [PubMed]

27. Folin, O.; Ciocalteu, V. On tyrosine and tryptophane determinations in proteins. J. Biol. Chem. 1927, 73, 627-650. [CrossRef]

28. Abuelizz, H.A.; Anouar, E.; Marzouk, M.; Taie, H.A.A.; Ahudhaif, A.; Al-Salahi, R. DFT study and radical scavenging activity of 2-phenoxypyridotriazolo pyrimidines by DPPH, ABTS, FRAP and reducing power capacity. Chem. Pap. 2020, 74, 2893-2899. [CrossRef]

29. Prasad, V.K.; Kavita, N.Y.; Chandrashekhar, L.B.; Manohar, J.P. Standardization and quantification of curcumin from Curcuma longa extract using UV visible spectroscopy and HPLC. J. Pharm. Phytochem. 2018, 7, 1913-1918.

30. Tavano, L.; Alfano, P.; Muzzalupo, R.; de Cindio, B. Niosomes vs. microemulsions: New carriers for topical delivery of capsaicin. Colloids Surf. B 2011, 87, 333-339. [CrossRef] [PubMed]

31. Sirk, T.W.; Brown, E.F.; Sum, A.K.; Friedman, M. Molecular dynamics study on the biophysical interactions of seven green tea catechins with lipid bilayers of cell membranes. J. Agric. Food Chem. 2008, 56, 7750-7758. [CrossRef] [PubMed]

32. Erlejman, A.G.; Verstraeten, S.V.; Fraga, C.G.; Oteiza, P.I. The interaction of flavonoids with membranes: Potential determinant of flavonoid antioxidant effects. Free Radic. Res. 2004, 38, 1311-1320. [CrossRef]

33. Phan, H.T.; Yoda, T.; Chahal, B.; Morita, M.; Takagi, M.; Mun'delanji, C.V. Structure-dependent interactions of polyphenols with a biomimetic membrane system. Biochim. Biophys. Acta 2014, 1838, 2670-2677. [CrossRef]

34. Tanaka, T.; Tamba, Y.; Masum, S.M.; Yamashita, Y.; Yamazaki, M. La ${ }^{3+}$ and Gd $\mathrm{Gd}^{3+}$ induce shape change of giant unilamellar vesicles of phosphatidylcholine. Biochim. Biophys. Acta 2002, 1564, 173-182. [CrossRef]

35. Tavano, L.; de Cindio, B.; Picci, N.; Ioele, G.; Muzzalupo, R. Drug compartmentalization as strategy to improve the physicochemical properties of diclofenac sodium loaded niosomes for topical applications. Biomed. Microdevices 2014, 16, 851-858. [CrossRef] [PubMed]

36. Lee, B.S.; Yuan, X.; Xu, Q.; McLafferty, F.S.; Petersen, B.A.; Collette, J.C.; John, S.Y. Preparation and characterization of antioxidant nanospheres from multiple $\alpha$-lipoic acid-containing compounds. Bioorg. Med. Chem. Lett. 2009, 19, 1678-1681. [CrossRef]

37. Patel, S.S.; Acharya, A.; Ray, R.S.; Agrawal, R.; Raghuwanshi, R.; Jain, P. Cellular and molecular mechanisms of curcumin in prevention and treatment of disease. Crit. Rev. Food Sci. 2020,60, 887-939. [CrossRef]

38. Naksuriya, O.; Okonogi, S. Comparison and combination effects on antioxidant power of curcumin with gallic acid, ascorbic acid, and xanthone. Drug Discov. Ther. 2015, 9, 136-141. [CrossRef] [PubMed]

39. AlBasher, G.; Abdel-Daim, M.M.; Almeer, R.; Ibrahim, K.A.; Hamza, R.Z.; Bungau, S.; Aleya, L. Synergistic antioxidant effects of resveratrol and curcumin against fipronil-triggered oxidative damage in male albino rats. Environ. Sci. Pollut. Res. 2020, 27, 6505-6514. [CrossRef]

40. Mansourizadeh, F.; Alberti, D.; Bitonto, V.; Tripepi, M.; Sepehri, H.; Khoee, S.; Crich, S.G. Efficient synergistic combination effect of Quercetin with Curcumin on breast cancer cell apoptosis through their loading into Apo ferritin cavity. Colloids Surf. B 2020, 191, 110982. [CrossRef]

41. Moghtderi, H.; Sepehri, H.; Delphi, L.; Attari, F. Gallic acid and curcumin induce cytotoxicity and apoptosis in human breast cancer cell MDA-MB-231. BioImpacts 2018, 8, 185-194. [CrossRef]

42. Sahu, A.K.; Mishra, J.; Mishra, A.K. Introducing Tween-curcumin niosomes: Preparation, characterization and microenvironment study. Soft Matter 2020, 16, 1779-1791. [CrossRef]

43. Kotenkov, S.A.; Gnezdilov, O.I.; Khaliullina, A.V.; Antzutkin, O.N.; Gimatdinov, R.S.; Filippov, A.V. Effect of cholesterol and curcumin on ordering of DMPC bilayers. Appl. Magn. Reson. 2019, 50, 511-520. [CrossRef]

44. Falck, E.; Patra, M.; Karttunen, M.; Hyvönen, M.T.; Vattulainen, I. Impact of cholesterol on voids in phospholipid membranes. J. Chem. Phys. 2004, 121, 12676-12689. [CrossRef]

45. Mazzotta, E.; Rossi, C.O.; Muzzalupo, R. Different BRIJ97 colloid systems as potential enhancers of acyclovir skin permeation and depot. Colloids Surf. B 2019, 173, 623-631. [CrossRef] [PubMed]

46. Lombardo, D.; Kiselev, M.A.; Caccamo, M.T. Smart nanoparticles for drug delivery application: Development of versatile nanocarrier platforms in biotechnology and nanomedicine. J. Nanomater. 2019, 2019. [CrossRef] 